\title{
浅谈药品检验的质量控制
}

\author{
留梦甜 \\ 杭州海王生物工程有限公司 \\ DOI:10.32629/eep.v2i10.492
}

[摘 要] 为了能够更好的保证我国药品的安全, 需要做好药品检验质量控制工作。在药品生产过程中, 需要对药品说明中不确定因素进行监测, 及时发现不足之处, 并采取有针对性的措施对其加以改进, 全面提高药品的质量安全。同时药检人员要将提高药品质检作为已任, 全面保障药品 的安全。

[关键词] 药品检验; 质量控制; 实践措施

\section{1 药品检验质量控制的必要性}

近几年, 我国医疗事业迅猛发展存进了药品中了和数量的大幅度提升, 药品的质量检验工作更加重要。药品检验作为医药品从研发、生产到流入 市场的中间环节, 只有药品检验的质量达标, 才能将其投入市场和临床使 用。因此, 国家及医院设有专门的药品检验机构, 目的就是为了督促检测人 员对药品的各项质量性质进行认真细致的检查与检测。

药品检验过程复杂、影响因素较多, 是一项繁杂、细致的工作, 每个环 节的微小误差都有可能影响最终的检验结果。因此, 加强药品检验的质量 控制是很有要的, 这样才能确保药品检验的可靠性和安全性。特别是从我 新药研究的现状来看, 一直以仿制为主, 虽然仿制药品是国外已经上市的 药品, 其临床效果均已有明确的评价和结论, 但是中药与西药在成分、配比 和生产流程中存在诸多不同, 并且国内外的生产技术能力和制造工艺也存 在很多差异, 一旦药品质量不达标, 将对患者的身体健康造成严重影响。因 此, 药品检验的质量控制也是评定药品安全的一项重要指标。

\section{2 我国药品检验的现状}

药品在流入市场之前, 会经过多个流程, 药品检验就是其中一项非常 重要的工作, 起到了连接药品研发、生产, 以及流入市场环节的作用。相关 标准指出, 药品只有经过检验机构的全面检验, 保证药品的质量达到既定 的标准以后, 才能流入到市场, 供给人们使用。但是受到多种主客观因素的 影响, 目前我国的药品检验的质量控制存在一些亟需解决的问题, 比如: 第 一, 药品检验机构的工作人员专业能力和综合素质水平有高有低, 很多药 品检验人员的能力不足, 对药品检验没有一个全面、充分的认识, 因此对待 工作的态度不认真, 对工作中所需要的设备和检验方法不熟悉; 第二, 在我 国部分偏远或者贫穷地区, 对药品检验工作的投入不够, 造成检验机构的 相关设备不能技术更新, 与社会和时代脱节; 第三, 就现阶段而言, 药品的 研发技术和手段越来越先进, 但是对药品检验的相关标注却仍然停留在过 去, 没有及时更新, 造成药品检验的相关标准不适用于当前的检验工作。

\section{3 药品检验质量控制实践措施}

3. 1 提高工作人员的综合素质和能力

药品检验质量控制过程中, 工作人员的综合素质和能力起到了关键的 作用和影响, 因此, 检验机构应该认识到这一点, 进而从根源出发, 做药品 检验的工作, 提高质量, 在这一过程中, 应当做到: 发挥药物质量监督和管 理部门的领导作用, 对药品检验人员做好相关资质审查, 保证所有参与工 作的人员都持有相应的资格证件; 为了提高工作人员的整体素质和综合能 力, 需要按照国家以及行业标注对员工实施定期考核; 对员工进行必要的 知识培训, 使其了解和掌握各项设备的使用以及工作流程, 然后根据相应
的检验标准和要求记录药品检验的数据, 不断增强期工作的专业性。

3. 2 增加对药品检验工作的扶持和帮助

药品检验工作的顺利开展, 离不开经济的扶持和帮助, 经济基础决定 上层建筑, 经济投入也是保证药品检验质量的一项重要基础。另外, 药品检 验工作本身就是一项相对专业和复杂的过程, 因此工作具有一定的难度。为 了提高药品检验的质量, 地方政府有关部门可以增加对药品检验工作的扶 持, 提高资金投入, 从而为药品检验创造优良的环境和技术设备支持, 保证 工作的先进性, 从而为药品质量检验的发展创造出良好的条件。

3. 3 完善药品检验的相关标准

在药品检验质量控制的相关工作中, 技术标准对其影响很大, 现阶段, 由于我国多种因素的影响, 使得目前的药品检验质量控制的很多标注没有 一个统一的标准, 因此, 药品检验标准的完善工作已经成为了药品检验行 业中需要立即解决的问题之一, 更多行业专业人员对这方面工作的进程给 予了重视。构建完善规范化药品检验标准, 药品检验单位需要针对目前的 问题, 结合实际情况, 制定出具有针对性的解决措施, 从药品检验记录不全 面问题入手, 不断提高检验记录的准确度和真实度, 但是药品检验的记录 不能作为质量控制的参考依据。

3. 4 将记录环节加以规范

药品检验工作具有一定的复杂性, 为了加强药品检验的质量和结果的 准确性, 需要使用专业的设备和仪器, 相关机构的工作人员在使用设备之 前, 应当对设备仪器的操作和使用流程有一个全面的了解, 提高使用的熟 练度, 另外, 不同种类的药品有着不同的药理和不同的成分, 在检验的过程 中不能选择同一种方式, 需要根据药物的类型选择合适的检验方法, 将药品 检验环节的各项数据详细记录。除此之外, 药品检验完成以后还需要对相关 资料做好收集和整理的工作, 通过数据分析, 研究影响数据结果的因素。

\section{4 结语}

药品质量直接影响着人们的生命健康, 因此为了让人们的生命健康得 到保证, 药品检验人员必须严格根据检验程序、标准对其进行检验, 做好药 品检验质量控制的具体工作, 保证药品的质量达到具体标准。

\section{[参考文献]}

[1]窦红允. 探讨药品检验的质量控制以及措施[J]. 临床医药文献电子 杂志,2019,6(45):194+196.

[2]袁云. 药品检验的质量控制策略探究 [J]. 中国卫生标准管 理,2018,9(04):84-86.

[3] 张荣琴. 药品检验的质量控制以及措施研究 [J]. 北方药 学,2018,15(11):186-187. 